\title{
The R2R3 MYB transcription factor MdMYB30 modulates plant resistance against pathogens by regulating cuticular wax biosynthesis
}

\author{
Ya-Li Zhang, Chun-Ling Zhang, Gui-Luan Wang, Yong-Xu Wang, Chen-Hui Qi, Qiang Zhao, Chun-Xiang You, \\ Yuan-Yuan $\mathrm{Li}^{*}$ and Yu-Jin $\mathrm{HaO}^{*}$
}

\begin{abstract}
Background: The MYB transcription factor family is one of the largest transcriptional factor families in plants and plays a multifaceted role in plant growth and development. However, MYB transcription factors involved in pathogen resistance in apple remain poorly understood.

Results: We identified a new MYB family member from apple, and named it MdMYB30. MdMYB30 was localized to the nucleus, and was highly expressed in young apple leaves. Transcription of MdMYB30 was induced by abiotic stressors, such as polyethylene glycol and abscisic acid. Scanning electron microscopy and gas chromatographmass spectrometry analyses demonstrated that ectopically expressing MdMYB30 in Arabidopsis changed the wax content, the number of wax crystals, and the transcription of wax-related genes. MdMYB30 bound to the MdKCS1 promoter to activate its expression and regulate wax biosynthesis. MdMYB30 also contributed to plant surface properties and increased resistance to the bacterial strain Pst DC3000. Furthermore, a virus-based transformation in apple fruits and transgenic apple calli demonstrated that MdMYB3O increased resistance to Botryosphaeria dothidea. Our findings suggest that MAMYB30 plays a vital role in the accumulation of cuticular wax and enhances disease resistance in apple.
\end{abstract}

Conclusions: MdMYB30 bound to the MdKCS1 gene promoter to activate its transcription and regulate cuticular wax content and composition, which influenced the surface properties and expression of pathogenesis-related genes to resistance against pathogens. MdMYB30 appears to be a crucial element in the formation of the plant cuticle and confers apple with a tolerance to pathogens.

Keywords: Apple, Cuticular wax, MdMYB30, Pathogens resistance

\section{Background}

Apple (Malus $\times$ domestica) is an important fruit crop that is commonly grown worldwide. The gloss and smoothness on apple are important traits that determine the market value of apple, as disease-free shiny fruits are more attractive to consumers. Cuticular wax is responsible for resistance to apple pathogens and gloss.

\footnotetext{
* Correspondence: liyy0912@163.com; haoyujin@sdau.edu.cn National Key Laboratory of Crop Biology; Shandong Collaborative Innovation Center of Fruit \& Vegetable Quality and Efficient Production; College of Horticulture Science and Engineering, Shandong Agricultural University, Tai-An 271018, Shandong, China
}

The plant cuticular structure is composed of two parts based on physical location: the cutin, which is close to the cell wall, and the epicuticular wax, which is exposed to the air. An epicuticular film of wax crystals covers the plant surface [1]. Cuticular wax plays crucial roles protecting against external environmental stress, such as acting as a transpiration barrier and functioning in interactions with pathogens. The basic constituents of plant cuticular wax are very long chain fatty acids (VLCFAs) and their derivatives. The cuticular synthetic pathway can be divided into three reactions: (1) de novo synthesis of C16 and C18 fatty acids; (2) extension of VLCFAs:

(c) The Author(s). 2019 Open Access This article is distributed under the terms of the Creative Commons Attribution 4.0 International License (http://creativecommons.org/licenses/by/4.0/), which permits unrestricted use, distribution, and 
the $\mathrm{C} 16$ and $\mathrm{C} 18$ fatty acids produced during the first stage extend on the endoplasmic reticulum to form C20-C36 VLCFAs; and (3) synthesis of derivatives of VLCFA, such as aldehydes, alcohols, alkanes, ketones, and esters [2].

The function of epicuticular wax as a transpiration barrier has been widely investigated. In maize, removing the wax layer on the surface of wild maize promotes the formation of adherent cells of rust on the leaf surface [3]. In addition, the epidermal wax on the surface of the leaf can be used to defend against natural enemies [4]. Some lipids may act as signaling substances during cell apoptosis and defend against external invasion [5].

MYB TFs are common among all eukaryotes; however, this protein family is particularly large in higher plants. The MYB protein family in animals contains three MYB conserved domain repeats (R1, R2, and R3), whereas most plant MYB TFs belong to the R2R3 type, which are associated with growth regulatory processes [6]. MYB proteins are reportedly related to a series of functions regulating secondary metabolism, such as epidermal wax [5], morphogenesis [7], and the abiotic and biotic stress responses $[8,9]$. The functions of the MYB family members have been widely studied. Overexpressing MYB94 increases total wax load approximately two-fold in Arabidopsis leaves, as MYB94 binds to the promoters of wax-related genes to enhance their expression [10]. Programmed cell death is a hypersensitive response that is closely related to plant disease resistance. AtMYB30 is an active modulator of cell death in both avirulent and virulent pathogen attack responses [11]. MYB30, MYB55, and MYB110 are three MYB proteins that are transcriptionally induced by microbe-associated molecular pattern (MAMP) treatment, which enhance resistance to fungal and bacterial pathogens and have important roles in rice plant immunity [12]. Furthermore, AtMYB30 shows great response to reactive oxygen species (ROS) and inhibits root cell elongation. This process involves multiple genes associated with VLCFA transport; thus, providing a molecular link between ROS - root cell - wax biosynthesis - plant immune responses [13].

The cuticle of fruit crops supplies morphological support for the integrity of the entire fruit and also affects growth and maturation [14]. The composition and content of cuticular wax may lead to differences in quality characteristics, such as post-harvest resistance to water loss, pathogen infection, and cracked fruit [15-17]. A large number of studies on fruit wax have concentrated on wax morphology and biosynthesis, whereas little is known about the molecular pathways regulating wax biosynthesis and their roles in apple. Here, we identified an apple R2R3 MYB TF named MdMYB30. Furthermore, ectopically expressing $M d M Y B 30$ improved disease resistance by changing the composition and surface properties of the wax. These results demonstrate that MdMYB30 positively regulates wax biosynthesis and acts as a pathogen resistance mechanism.

\section{Results \\ Identification of the MYB30 gene in apple}

To separate the MdMYB30 gene, the AtMYB30 gene was used as bait to search sequences in apple based on homologous retrieval. We chose a gene with the highest homology to AtMYB30, and named it MdMYB30 (MDP0000149102). The MdMYB30 cDNA contained two noncoding regions (Additional file 1: Figure S1), and the MdMYB30 ORF encoded a 342 amino acid polypeptide as predicted by DNAMAN 6.0 software [18]. We analyzed the amino acid sequences of MdMYB30 and AtMYB30. Figure 1a shows that the MdMYB30 and AtMYB30 protein sequences were highly similar and contained conserved R2R3 domains.

A phylogenetic tree was constructed to investigate the evolutionary relationship of MYB30s among species (Fig. 1b; Additional file 1: File S1). The results indicated that apple MdMYB30 exhibited the closest evolutionary relationship with pear PbMYB30 (XP_009376268), because they were in the same clade. MdMYB30 had the most distant relationship with maize ZmMYB30 (XP 008652703.1), wheat TaMYB30 (AYK27536.1), and rice OsMYB30 (BAT05602.1), as all three are monocotyledonous plants. The phylogenetic tree indicated a clear boundary between dicotyledons and monocotyledons.

\section{MdMYB30 is localized in the nucleus and is induced by abiotic stress}

The localization of a protein is very important for its function. Figure $2 \mathrm{a}$ indicates that the green fluorescent signal and the blue fluorescent signal merged in the nucleus, demonstrating that MdMYB30 localized to the nucleus. The $M d M Y B 30$ transcription analysis in various apple tissues showed that although it was constitutively expressed in all examined tissues, the expression levels were different, indicating the specific function of $M d M Y B 30$ in its highly expressed tissues (Fig. 2b).

To begin to investigate the $M d M Y B 30$ stress response, cis-elements in the $M d M Y B 30$ promoter were generated by applying PlantCARE software [19]. A number of regulatory sequences in the $M d M Y B 30$ promoter were identified, including elements involved in heat, low-temperature, and drought stressors, elements involved in plants, such as SA, $\mathrm{ABA}$, and $\mathrm{GA}$, and several light-responsive elements (Table 1), suggesting that MdMYB30 is closely related to plant response to various abiotic stresses.

Subsequently, we analyzed the expression of the MdMYB30 gene treated with osmotic stress (PEG), ABA, $\mathrm{GA}$, or $\mathrm{NaCl}$, respectively (Fig. 2c). In vitro-propagated apple seedlings were used for this experiment. The 

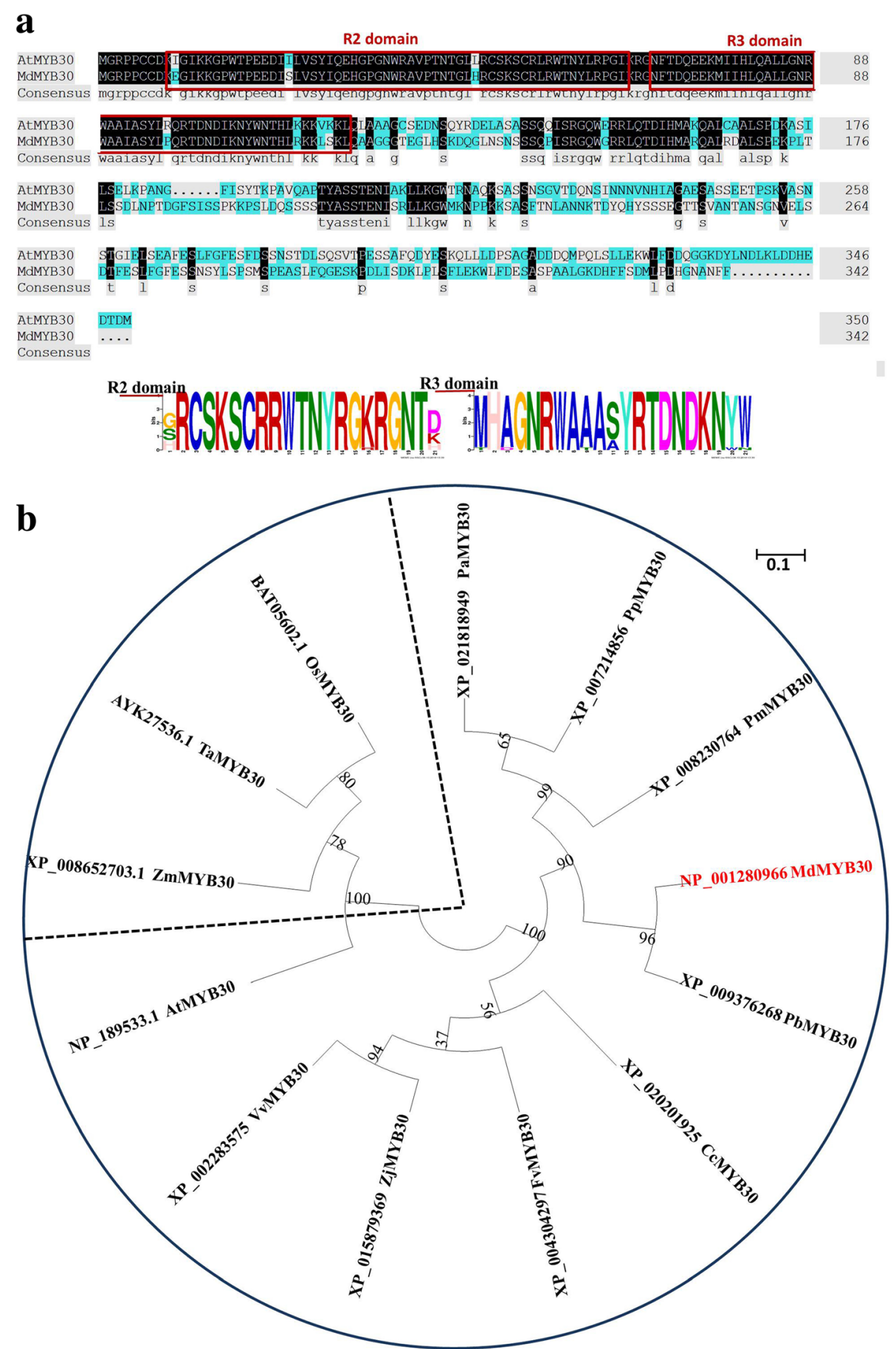

Fig. 1 Sequence alignment and phylogenetic analysis of MdMYB30. a Alignment of the MdMYB30 and AtMYB30 amino acid sequences. The locations of two conserved motifs are labeled with red lines. The conserved R2 and R3 domains required for DNA binding of MdMYB30 and AtMYB30 proteins. Conservation of residues across the MdMYB30 and AtMYB30 proteins is indicated by the height of each letter. Bit scores indicate information for each conserved motif in the sequence (To interpret the colors in this legend, please refer to the web version of this article). $\mathbf{b}$ Phylogenetic relationship analysis of the plant MYB30 proteins. Phylogenetic analysis of MdMYB30 and 12 other plants MYB30 protein sequences obtained from the NCBI database. MdMYB30 is denoted in red font, and the scale bar indicates the branch length. MdMYB30: NP_001280966 Malus domestica; PbMYB30: XP_009376268 Pyrus bretschneideri; PmMYB30: XP_008230764 Prunus mume; PaMYB30: XP_021818949 Prunus avium; PpMYB30: XP_007214856 Prunus persica; FvMYB30: XP_004304297 Fragaria vesca; ZjMYB30: XP_015879369 Ziziphus jujube; VvMYB30: XP_002283575 Vitis vinifera; CcMYB30: XP_020201925 Cajanus cajan; TaMYB30: AYK27536.1 Triticum aestivum; OsMYB30: BAT05602.1 Oryza sativa; ZmMYB30 XP_008652703.1 Zea mays; AtMYB30:NP_189533.1 Arabidopsis thaliana 


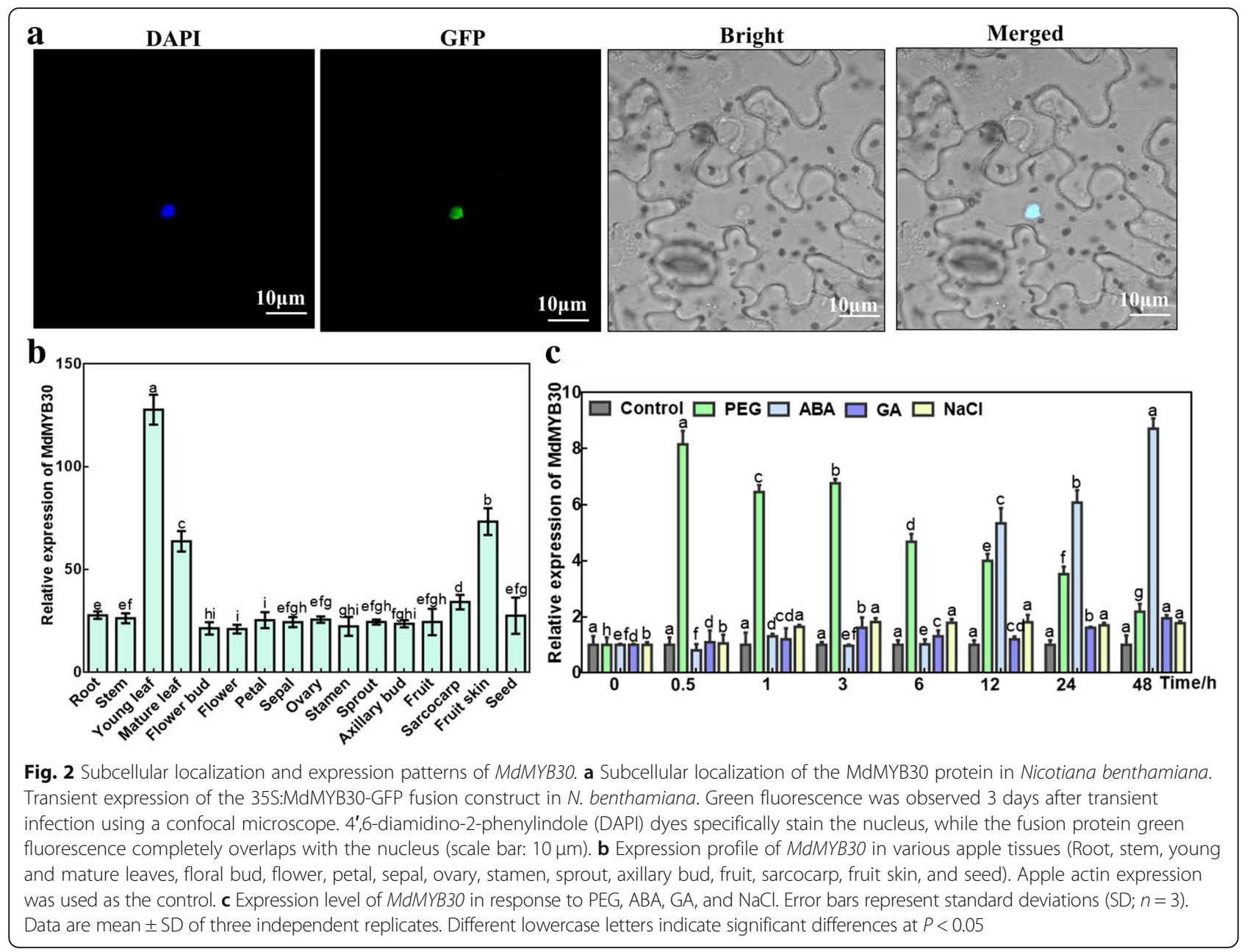

Table 1 Promoter cis-acting element analysis of MdMYB30

\begin{tabular}{lll}
\hline Regulatory sequence & sequence & Function of site \\
\hline ABRE & GCCGCGTGGC & cis-acting element involved in the abscisic acid responsiveness \\
ACE & AAAACGTTTA & cis-acting element involved in the abscisic acid responsiveness \\
ARE & AAAACGTTA & cis-acting regulatory element essential for the anaerobic induction \\
G-box & CACGAC & cis-acting regulatory element involved in light responsiveness \\
GARE-motif & TCTGTTG & gibberellin-responsive element \\
GATA-motif & GATAGGG & part of a light responsive element \\
HSE & AAAAAATTC & cis-acting element involved in heat stress responsiveness \\
LAMP-element & CTTATCA & part of a light responsive element \\
LTR & CCGAAA & cis-acting element involved in low-temperature responsiveness \\
MBS & CAACTG & MYB binding site involved in drought-inducibility \\
P-box & CCTTTTG & gibberellin-responsive element \\
SP1 & CC(G/A)CCC & light responsive element \\
TC-rich repeats & ATTTCTCCA & cis-acting element involved in defense and stress responsiveness \\
TCA-element & GAGAAGAATA & cis-acting element involved in salicylic acid responsiveness \\
\hline
\end{tabular}


MdMYB30 transcript level increased rapidly peaking at 0.5 -h, and then gradually declined in the PEG treatment. $M d M Y B 30$ expression did not change during the early stage in response to ABA, but increased sharply after 12-h of treatment and reached its maximum at 48-h. No changes in the MdMYB30 transcript levels were detected after the $\mathrm{GA}$ and $\mathrm{NaCl}$ treatments.

\section{MdMYB30 functions in biotic and abiotic stress}

We transformed MdMYB30 into wild-type Arabidopsis to investigate the role of $M d M Y B 30$ during stress. Six independent MdMYB30 ectopically expressing (MdMYB30 OE) Arabidopsis lines as determined by qPCR were obtained. MdMYB30 OE4, OE5, and OE6 with higher MdMYB30 transcription levels were selected for subsequent experiments
(Fig. 3a). We monitored the flg22-induced immune response, PEG-induced osmotic stress, and ABA sensitivity of $M d M Y B 30$ to ectopic expression in Arabidopsis. No clear differences were detected between WT and MdMYB30 ectopic expression Arabidopsis in normal MS medium. $M d M Y B 30$ ectopic expression roots were longer under the flg22 or PEG treatment than those in WT. However, the growth of roots in MdMYB30 ectopic expression was significantly inhibited under ABA treatment, compared to the WT. The change in root elongation was more obvious with the increase of flg22, PEG, and ABA concentrations (Fig. 3b, c). Fresh weight and chlorophyll in the MdMYB30 ectopic expression lines improved significantly after the flg22 and PEG treatments, whereas they decreased with the ABA treatment, compared to the WT, indicating that ectopic expression of

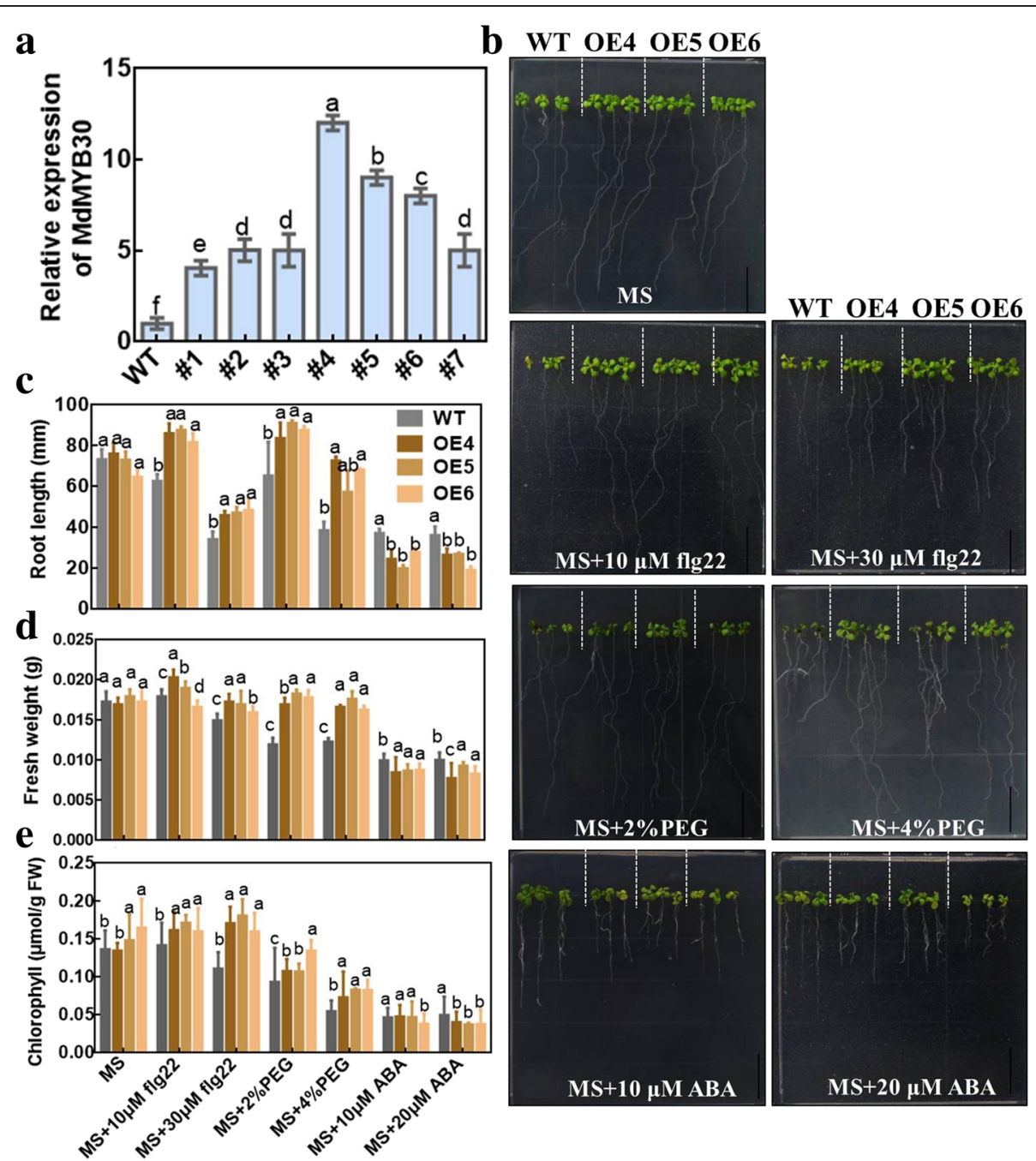

Fig. 3 MdMYB30 participates in the biotic and abiotic stress response. a Identification of ectopically expressed Arabidopsis thaliana expression level of MdMYB30 by qRT-PCR. b Phenotype of wild-type and MdMYB30 ectopically expressed Arabidopsis treated with or without $10 \mu \mathrm{M}$ flg22, $30 \mu \mathrm{M}$ flg22, 2\% PEG, 4\% PEG, $10 \mu \mathrm{M}$ ABA, or $20 \mu \mathrm{M}$ ABA (scale bar: $1 \mathrm{~cm}$ ). c-e Root length $\mathbf{c}$, fresh weight $\mathbf{d}$, and chlorophyll content $\mathbf{e}$ in $\mathbf{b}$ Arabidopsis seedlings. Arabidopsis chlorophyll $\mu \mathrm{mol} / \mathrm{g}$ FW (fresh weight $=\mathrm{FW}$ ). Error bars represent standard deviation $(S D ; n=3)$. Data are mean \pm SD of three independent replicates. Different lowercase letters indicate significant differences at $P<0.05$ 
the MdMYB30 lines increased resistance to biotic stress, osmotic stress, and improved sensitivity to ABA (Fig. 3c-e). Therefore, these data suggest that $M d M Y B 30$ participates in the response to biotic and abiotic stress.

\section{MdMYB30 alters epicuticular wax load and composition}

Previous studies have demonstrated that AtMYB30 is an important regulator of wax biosynthesis in Arabidopsis [20]. Total wax of $M d M Y B 30$ ectopic expression and WT Arabidopsis was extracted to study the function of MdMYB30 in wax accumulation. A significant difference was found in the total wax load between ectopically expressing MdMYB30 and WT Arabidopsis. The total wax load was 1.5- to 1.3-fold higher in the stems and leaves of the MdMYB30 OE lines compared to WT plants (Fig. 4a; Additional file 1: Figure S2a; Additional file 1: Table S1). Then, GC-MS was applied to determine the stem wax components. The analysis of cuticular wax composition showed that the contents of alkanes, alcohols, aldehydes, fatty acids, ketones, and esters increased in the MdMYB30 ectopic expression lines compared with the WT. Among them, the increase in fatty acids was the largest, which was more than 2.6-fold higher (Fig. 4b; Additional file 1: Table S2). C29 alkanes, C31 alcohols, C29 aldehydes, C16 fatty acids, C29 ketones, and C29 and C30 esters increased significantly in the $M d M Y B 30$ ectopic expression lines compared with those in the WT, providing evidence that MdMYB30 plays an

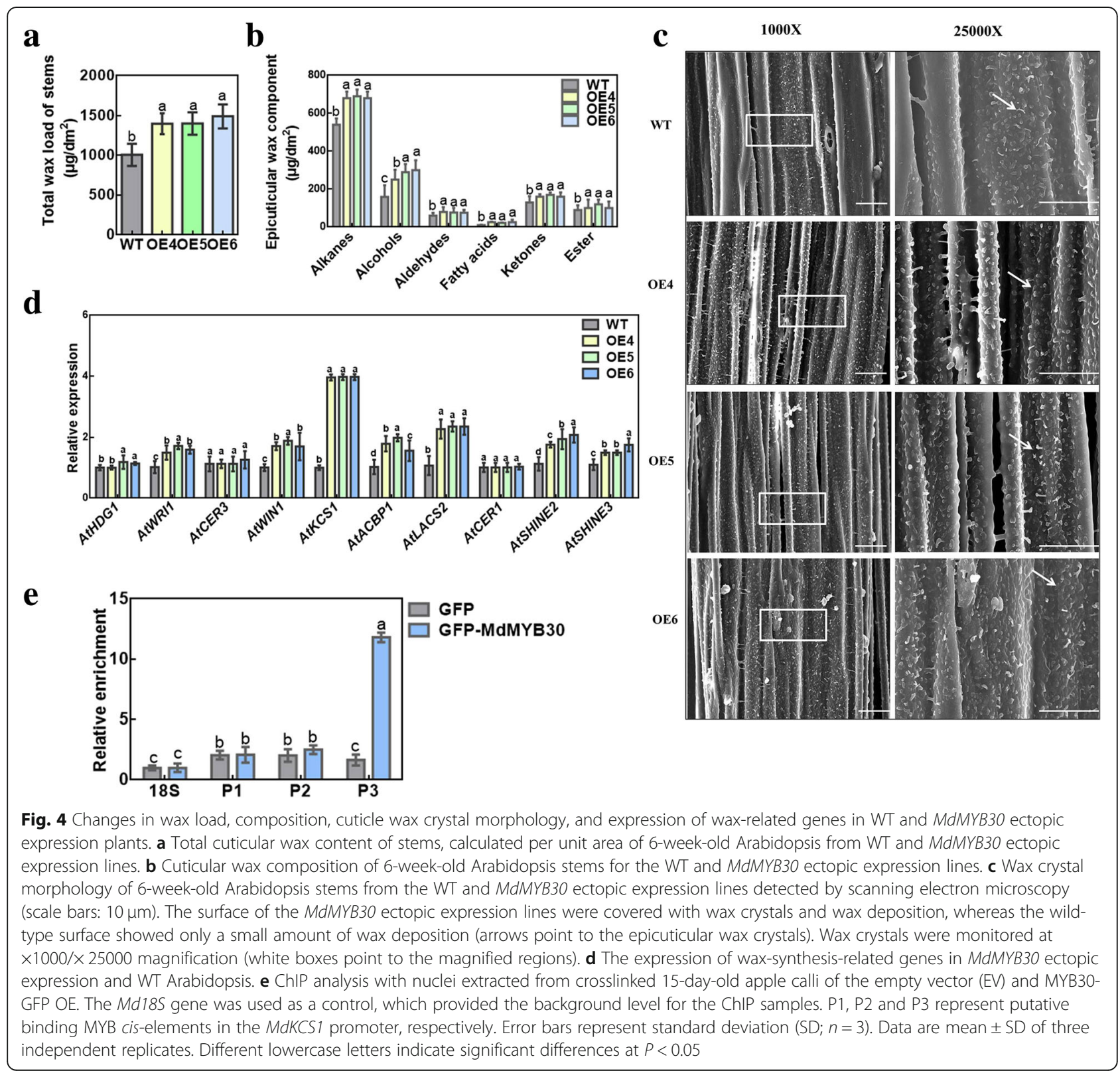


important role in cuticular wax biosynthesis (Additional file 1: Figure S2b).

We examined wax crystal morphology in the stems of MYB30 ectopically expressing and WT plants, but did not find a difference in wax crystal morphology using SEM; however, the MdMYB30 ectopically expressing Arabidopsis exhibited more epicuticular wax crystals than the WT (Fig. 4c). Similarly, the wax crystals in ectopically expressing Arabidopsis leaves were also examined, and just a small increase was detected (Additional file 1: Figure S2c). Therefore, expression of MdMYB30 changed the numbers of wax crystals in stems and leaves.

Furthermore, the transcription levels of wax biosynthesis genes, including AtHDG1, AtWRI1, AtCER3, AtWIN1, AtKCS1, AtACBP1, AtLACS2, AtCER1, AtSHINE2, and AtSHINE3 in the WT and MdMYB30 ectopically expressed lines were detected by qRT-PCR. Ectopic expression of MdMYB30 upregulated the transcription of several wax-related genes, such as AtWRI1, AtWIN1, AtKCS1, AtACBP1, AtLACS2, AtSHINE2, and AtSHINE3, indicating that $M d M Y B 30$ promotes wax biosynthesis (Fig. 4d). Interestingly, AtKCS1 was significantly upregulated in MdMYB3O ectopic expression plants. Therefore, we analyzed the $M d K C S 1$ promoter sequence and found three motifs (P1, P2, and P3) that might interact with the MdMYB30 protein (Additional file 1: Figure S3). The ChIP-PCR assay was carried out to examine if MdMYB30 directly bound to the MdKCS1 promoter. The results showed that MdMYB30 specifically bound to the TAATTT motif in the P3 sequence of the MdKCS1 promoter to regulate wax synthesis (Fig. 4e).
MdMYB30 functions to change epicuticular wax properties and cuticular permeability

The thickness of the wax layer is associated with cuticular permeability. The chlorophyll leaching assay was performed to investigate whether cuticular membrane properties in MdMYB30 ectopically expressing plants changed. The results indicated that chlorophyll was extracted at a slower rate from $M d M Y B 30$ ectopically expressing plants compared to WT (Fig. 5a), suggesting higher cuticular resistance for chlorophyll leaching in the ectopically expressing Arabidopsis. Subsequently, a toluidine blue staining experiment suggested that the MdMYB30 ectopically expressing lines were more difficult to stain than the WT, regardless of whether rosette leaves or stems were stained (Fig. 5b, c). These results indicate that MdMYB30 notably changed the permeability of the cuticular wax.

\section{MdMYB30 confers resistance to Pst DC3000}

Cuticular wax acts as both a physical barrier against stressors and pathogens and is a chemical deterrent and activator of the plant defense response. We assessed whether MdMYB30 affected resistance to Pst DC3000, which is a kind of bacterial pathogen. The results showed that WT leaves exhibited yellowing after 3 days of inoculation with Pst DC3000; however, the leaves of the MdMYB30 ectopically expressing lines showed little yellowing. Susceptibility became more and more serious through time, and almost all WT rosette leaves developed chlorosis after $13 \mathrm{~d}$ of infection, whereas the leaves of the ectopically expressed lines were healthier at all time points examined, suggesting that $M d M Y B 30 \mathrm{ec}-$ topic expression conferred resistance to the pathogen
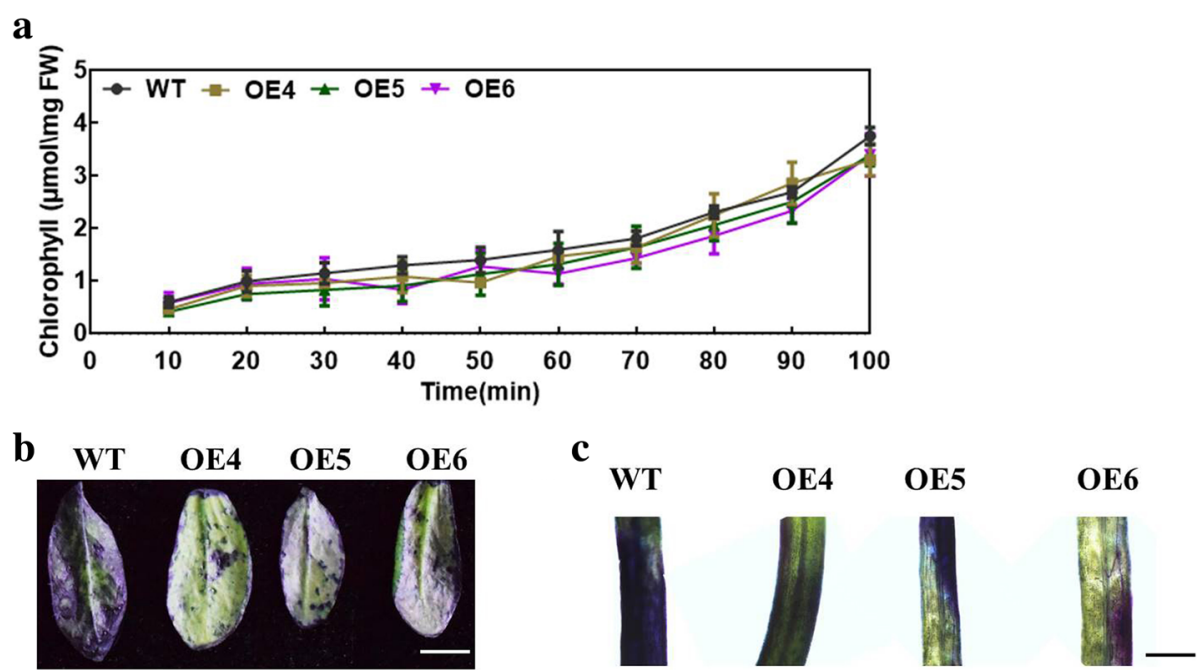

Fig. 5 Leaf surface permeability of WT and MdMYB30 ectopic expression plants. a Chlorophyll leaching rates in mature rosette leaves of WT and MdMYB30 ectopic expression Arabidopsis chlorophyll $\mu \mathrm{mol} / \mathrm{mg}$ FW (fresh weight = FW). b and $\mathbf{c}$ Toluidine blue staining of WT and MdMYB30 ectopic expression Arabidopsis leaves $\mathbf{b}$ (scale bars: $1 \mathrm{~cm}$ ) and stems c (scale bars: $500 \mu \mathrm{m}$ ) 
(Fig. 6a). ROS levels are closely associated with a pathogen infection. To test whether the ectopically expressing lines had altered ROS levels after inoculation with Pst DC3000 for $5 \mathrm{~d}$, the ROS reactive dyes DAB and NBT were used to test $\mathrm{O}_{2}{ }^{-}$and $\mathrm{H}_{2} \mathrm{O}_{2}$ contents in infected rosette leaves. The results showed that ectopically expressing MdMYB30 stained strongly, whereas weak staining was observed in the WT (Fig. 6c-f). Correspondingly, callose content improved in the ectopically expressing lines after the Pst DC3000 infection compared to the WT (Fig. 6b), demonstrating that $M d M Y B 30$ enhanced resistance to Pst DC3000.

\section{MdMYB30 positively regulates $B$. dothidea attack via transient transformation in apple fruits}

The expression of $M d M Y B 30$ was increased or repressed using viral vector-based transient transformation to confirm the function of $M d M Y B 30$ in enhancing resistance against pathogens in apple. Here, independent MdMYB30-TRV and MdMYB30-pIR transient expression of MdMYB30

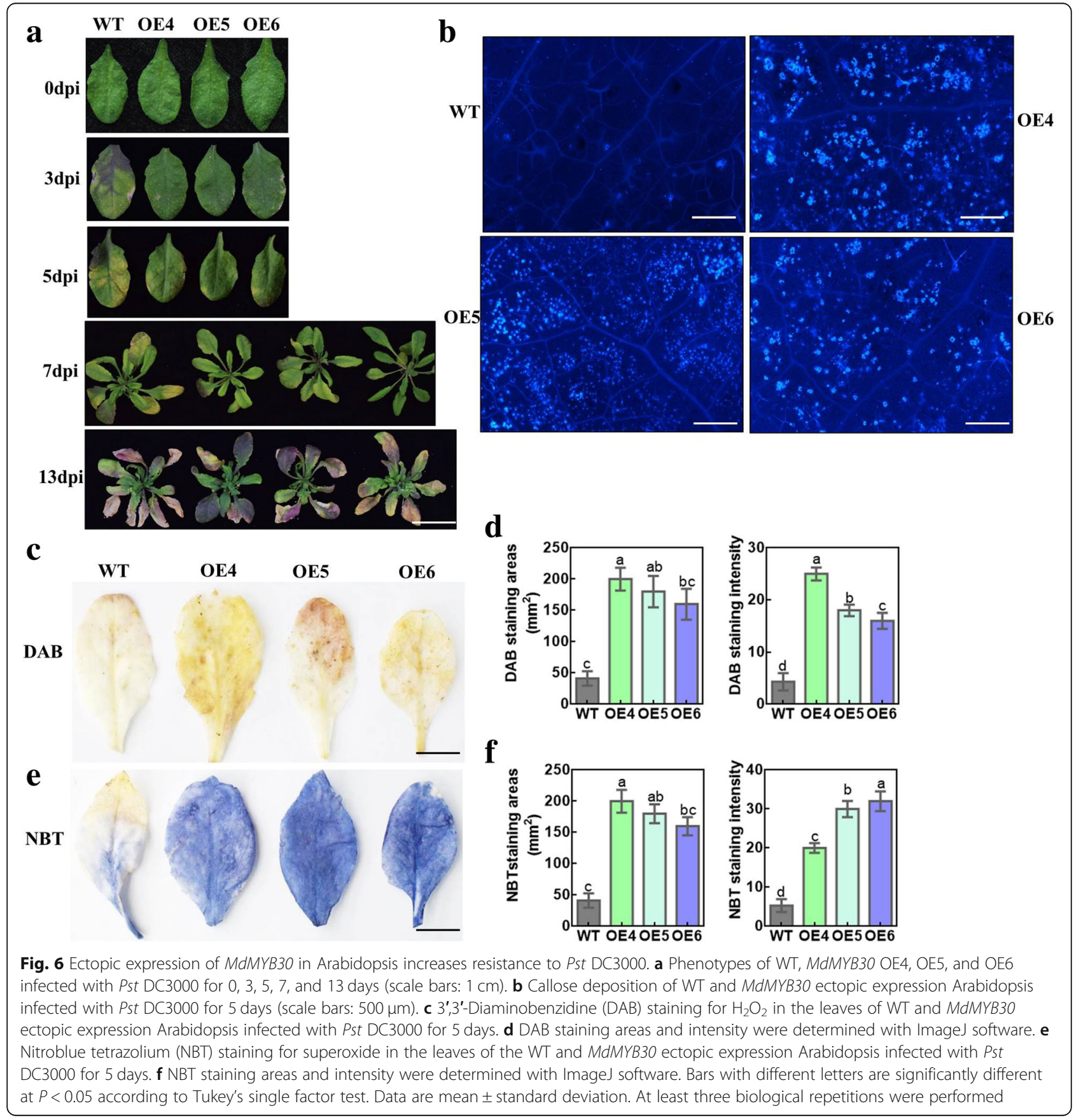


mRNA levels was downregulated or significantly upregulated, respectively (Fig. 7a). First, we detected the transcription of wax-related genes at the injection point, and three wax-related genes were positively correlated with MdMYB30 expression (Fig. 7b). Furthermore, overexpression of MdMYB30 accelerated the numbers of wax crystals around the injection sites according to SEM, compared to the control (Fig. 7c). Correspondingly, overexpression of $M d M Y B 30$

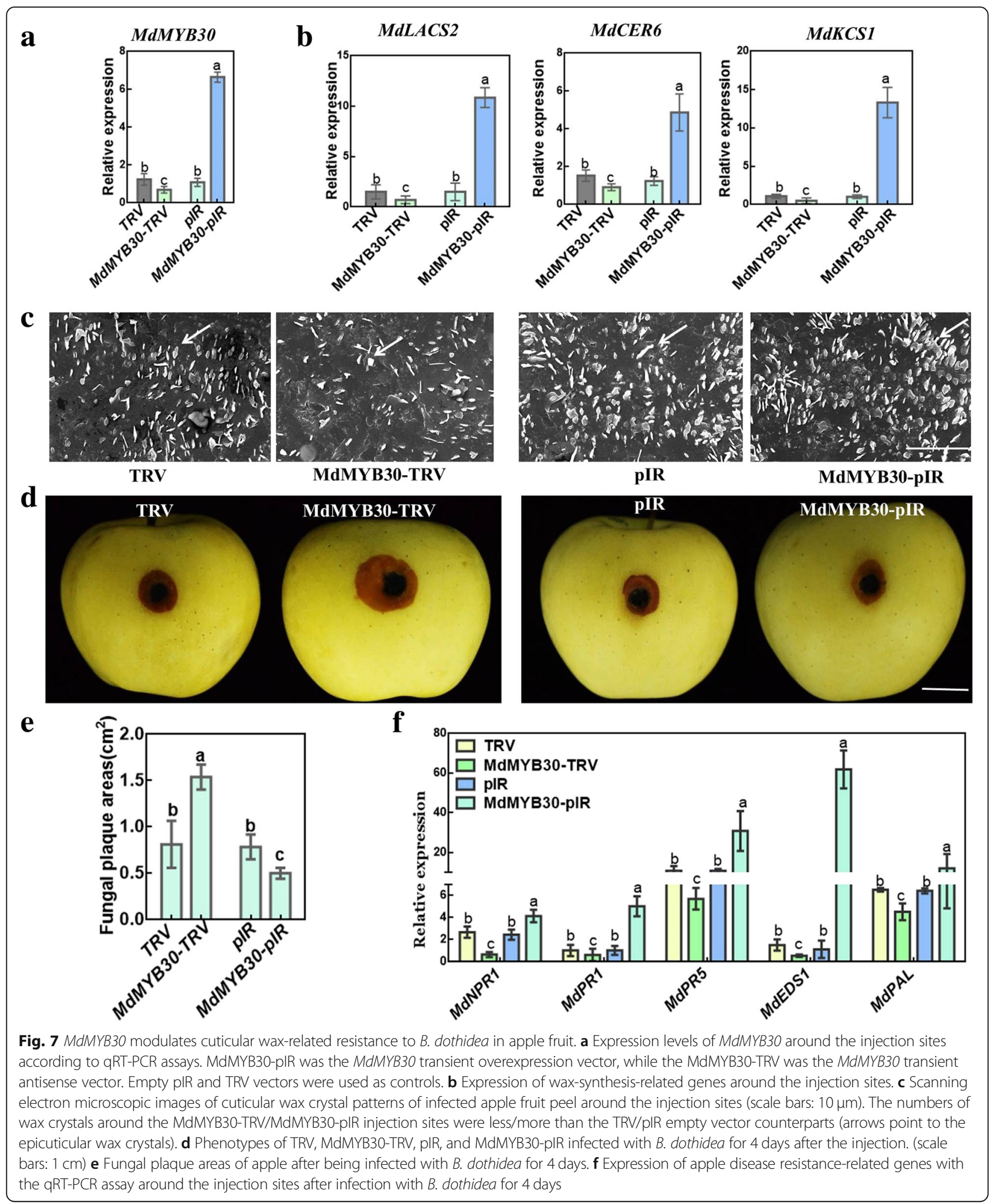


decreased significantly, while its suppression increased the lesions, compared to the empty vector (Fig. 7d, e). The qRTPCR results demonstrated that transcription of pathogenesisrelated genes, including $M d N P R 1, M d P R 1, M d P R 5$, MdEDS1, and MdPAL decreased at the TRV-MdMYB30 injection sites and increased at the pIR-MdMYB30 injection sites compared with the controls (Fig. 7f). Subsequently, the function of $M d M Y B 30$ in disease resistance was confirmed in apple calli. We overexpressed $M d M Y B 30$ in apple calli and obtained three MdMYB30 overexpressing (MdMYB30 OX) transgenic lines (Fig. 8a). Then, we tested the immunity of apple calli to $B$. dothidea, and found that overexpressing $M d M Y B 30$ notably reduced the lesions compared to that of the empty vector (Fig. 8b, c). MdMYB30 OX transgenic apple calli had higher levels of $\mathrm{H}_{2} \mathrm{O}_{2}$ than Empty Vector (Fig. 8d). These findings demonstrate that MdMYB30 positively regulates wax accumulation in apple fruit, and strengthened apple resistance to $B$. dothidea.

\section{Discussion}

Epicuticular wax participates in many physiological processes and is the main protective substance in the aerial parts of plants. MYB TFs participated in numerous processes [6, 21, 22]. Several R2R3 type MYB TFs play essential roles in biological stress through a possible cuticular-dependent pathway [23, 24], but the role of R2R3-MYB proteins in disease resistance is poorly understood in apple.

Protein subcellular localization is closely related to its function. It was reported that many MYB proteins are localized in the nucleus. In wheat (Triticum aestivum L.), TaMYB1 is localized in the nucleus as indicated by an in vivo subcellular targeting experiment in onion epidermal cells [25]. In Arabidopsis, MYB21 and MYB24 are localized in the nucleus where they interact with $M Y C 2 / 3 / 4 / 5$ proteins to modulate stamen growth and seed production through a bHLH-MYB complex [26]. The nuclear localized transcription factor MYB2 interacts with bHLH proteins in Apium graveolens to regulate anthocyanin biosynthesis [27]. In this study, the MdMYB30 fluorescence signal indicated that it was located in the nucleus, demonstrating that MdMYB30 as a transcription factor (TF) might target the genes localized in the nucleus that are involved in wax synthesis.

Plant MYB TFs play important roles in regulatory pathways and networks facing various stressors. In $L$. purpureus, LpMYB1 ectopically expressing Arabidopsis exhibits enhanced drought and salt resistance compared to WT [28]. In Arabidopsis, AtMYC2 and/or AtMYB2 overexpressing plants show higher sensitivity to ABA and several ABA-inducible genes are upregulated, indicating that both proteins transcriptionally activate the expression of the ABA-inducible gene under drought stress [22]. In our study, ectopic expression of $M d M Y B 30$ in Arabidopsis improved the responses to abiotic (PEG and ABA) and biotic (flg22 and B. dothidea) stressors.

Previous studies have shown that cuticular wax is mainly composed of alkanes, alcohols, aldehydes, free acids, ketones, and esters. Among them, alkanes account a

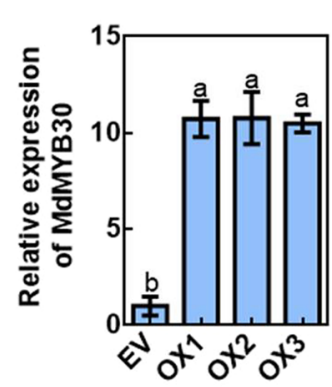

b

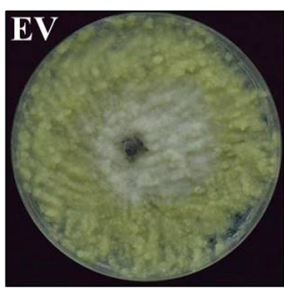

c

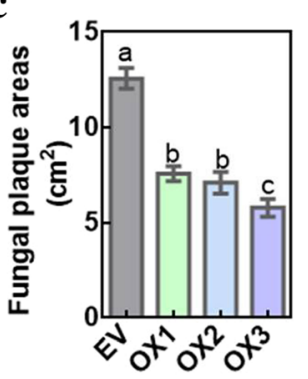

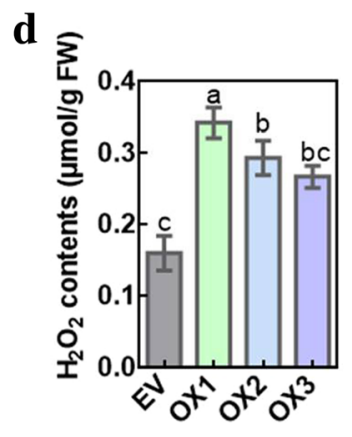
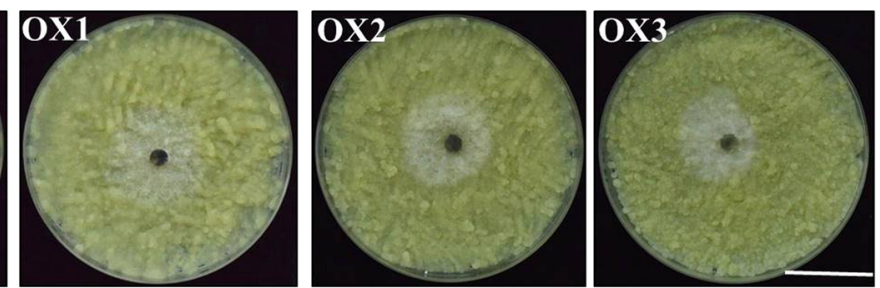

Fig. 8 Overexpressing MdMYB30 in apple calli increases resistance to B. dothidea. a Identification of transgenic apple calli expression level of MdMYB30 by qRT-PCR. $\mathbf{b}$ Phenotypes of EV, MdMYB3O OX1, OX2, and OX3 calli infected with B. dothidea for 4 days (scale bars: $1 \mathrm{~cm}$ ). $\mathbf{c}$ Determination of fungal plaque areas of EV, MdMYB30 OX1, OX2, and OX3 calli infected with $B$. dothidea for 4 days. $\mathbf{d} \mathrm{H}_{2} \mathrm{O}_{2}$ contents of EV, MdMYB30 OX1, OX2, and OX3 calli infected with $B$. dothidea for 4 days. Bars with different letters are significantly different at $P<0.05$ according to Tukey's single factor test. Data are mean \pm standard deviation. At least three biological repetitions were performed 
for $40-60 \%$, alcohols account for $15-20 \%$, aldehydes account for $5-10 \%$, free acids account for $5-9 \%$, ketones account for $15-20 \%$, and esters account for $<10 \%$ of wax content. Increased alkane content in Arabidopsis has the greatest impact on total wax quality [29-31]. Our results show that wax content of each component is consistent with previous ones and the most obvious increase in wax content was fatty acids, compared to WT. We suspect that fatty acids may play a role in disease resistance of the plant epidermis. Studies of Arabidopsis long-chain acyl-CoA synthetase2 (LACS2) mutants have demonstrated that modifications in epidermal wax component, structure, and permeability may result in strong resistance to Botrytis cinerea [32]. An increase in wax content usually causes changes in the crystalline structure and quantity of wax crystals [33, 34]. Here, epidermal wax load and the number of wax crystals increased in MdMYB30 ectopic expression Arabidopsis, which was possibly due to altered epidermal wax composition.

Previous reports indicate that the AtKCS1, AtFDH, and $A t D H 3$ genes are involved in VLCFA biosynthetic pathways. MYB30 directly activates the promoters of these genes to modulate cell death by increasing the biosynthesis of VLCFAs in the endoplasmic reticulum [5]. Transcription of the cuticular wax-related gene MYB96 was induced by drought and $\mathrm{ABA}$ and specifically bound to the promoter regions of several structural genes associated with wax biosynthesis. The increased wax content subsequently leads to enhanced drought tolerance in plants [35]. Our results demonstrate that enzyme-encoding genes and TFs increased significantly in MdMYB30 ectopic expression Arabidopsis compared to WT. Among them, AtKCS1 was the most obvious, which is consistent with $M d M Y B 30$ binding to the $M d K C S 1$ promoter, thereby activating its expression.

As toluidine blue directly enters plant tissues without a solvent, this method can be used to study permeability of the cuticular membrane [32]. We used the toluidine blue staining assay to determine epidermal wax permeability. A previous study showed that Vicia faba leaves infected with bean yellow mosaic virus exhibit higher $\mathrm{H}_{2} \mathrm{O}_{2}$ and MDA levels compared to controls [36]. Here, the MdMYB30 transgenic apple calli and ectopically expressing Arabidopsis leaves exhibited more $\mathrm{O}_{2}{ }^{-}$and $\mathrm{H}_{2} \mathrm{O}_{2}$ contents than the WT after treatment with Pst DC3000 and B. dothidea for 4 days, respectively. Actually, a burst of ROS is considered one of the earliest responses to a biotic stress [37]. The ROS burst enhances plant disease resistance and increases changes in the cell walls [38]. These findings support previous views and confirm that $M d M Y B 30$ positively regulates plant disease resistance.

\section{Conclusions}

In conclusion, we present evidence that MdMYB30 improved plant disease resistance and we propose a working model to explain this process (Fig. 9). Briefly, the TF $M d M Y B 30$ regulates the biosynthesis of cuticular wax by activating the expression of $M d K C S 1$. Thus, ectopically expressing $M d M Y B 30$ significantly increased the total amount of cuticular wax and changed its composition; therefore, changing the surface properties and pathogenesis-related genes to resist against pathogens.

\section{Methods}

\section{Plant materials and growth conditions}

'Orin' apple calli (wild type) were cultured in the dark at $23-25^{\circ} \mathrm{C}$ and subcultured every 3 weeks. The 'Royal Gala' tissue culture seedlings were cultured at $23-25^{\circ} \mathrm{C}$ under a $16 \mathrm{~h} / 8 \mathrm{~h}$ photoperiod and subcultured once per month. The subcultured mediums used were followed by [39]. Arabidopsis (ecotype 'Columbia') were grown in a long-day $(16 \mathrm{~h} / 8 \mathrm{~h})$ light incubator at $19-22^{\circ} \mathrm{C}$. Tobacco (Nicotiana benthamiana) was grown at $23-25^{\circ} \mathrm{C}$ under $14 \mathrm{~h} / 10 \mathrm{~h}$ photoperiod.

Individual apple tissues were taken from 5-year-old 'Royal Gala' trees for the tissue expression experiments. The apple seedling treatment method for the stress response experiments followed that described previously [40]. The apple fruits were sampled 140 days after blooming from 'Golden Delicious' trees. B. dothidea was grown on potato dextrose agar (PDA) medium at $24{ }^{\circ} \mathrm{C}$ [41].

'Orin' apple calli was given by Prof. Takaya Moriguchi of the National Institute of Fruit Tree Science, Japan. 'Royal Gala' trees were obtained from the experimental station of Shandong Agricultural University. 'Golden Delicious' fruits were obtained from a commercial orchard

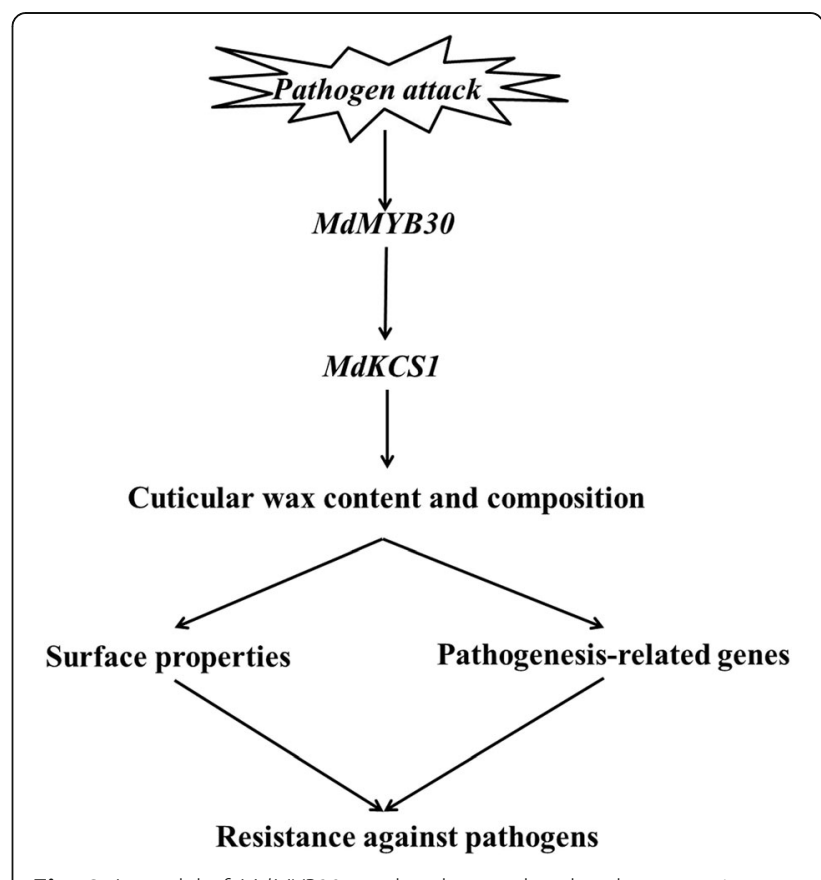

Fig. 9 A model of MdMYB30 regulated wax-related pathogen resistance 
near Tai-An City. We declare that the collection of plant materials comply with institutional, national, or international guidelines.

\section{Phylogenetic tree construction and amino-acid sequence analysis}

The homologs of Arabidopsis MYB30 were obtained from the Protein BLAST program (http://www.ncbi.nlm.nih. gov/BLAST/). The phylogenetic tree and multiple sequence alignments were made using MEGA 7.0 and DNAMAN 6.0 software, respectively. MEME Suite (http:// meme-suite.org/) software was used to obtain the functional motifs of the MYB30S. The MdMYB30 protein structural domain was predicted through NCBI website (https://www.ncbi.nlm.nih.gov/Structure/cdd/wrpsb.cgi).

\section{Plasmid construction and plant genetic transformation} Leaves of 'Royal Gala' tissue-cultured seedlings were used to extract RNA to clone MdMYB30 cDNA. The $1026 \mathrm{bp}$ MdMYB30 cDNA was amplified using the MdMYB30-PRIF (5'-GTCGACATGGGGAGGCCTCCTTGCT-3') and MdMYB30-PRI-R (5'-GGATCC GAAAAAGTTAGCATT TCCATGATCT-3') primers, designed with the SalI and BamHI restriction endonuclease sites, respectively. To obtain the MdMYB30-GFP fusion protein, the amplification product was inserted into PRI-GFP. Transformations of apple calli and Arabidopsis followed the methods described in $[42,43]$, respectively.

\section{Subcellular localization}

The full length MdMYB30 sequence was inserted into the GFP vector to construct the recombinant plasmid MdMYB30-GFP. 35S::MdMYB30-GFP, which was used to transform tobacco [44]. The fluorescent signals were detected using a laser scanning confocal microscope (Zeiss LSM 510 META, Jena, Germany).

\section{Wax extraction, gas chromatography-mass spectrometry} (GC-MS) analysis, and scanning electron microscopy (SEM) Cuticular wax was extracted exhaustively using the following steps: chloroform extraction, nitrogen blow drying, derivatization reaction, and sample analysis following that described previously [39, 45]. SEM was performed as described previously [39]. The steps can be summarized as: sampling, vacuum freeze drying, gold spray treatment, observation of the mirror.

\section{Chromatin immunoprecipitation (ChIP)-polymerase chain reaction (PCR) analysis}

The ChIP-PCR analysis was performed using the EpiTect ChIP OneDay Kit following the method described by [46]. The primers used in the ChIP-PCR analysis are listed in Additional file 1: Table S3.

\section{Gene expression analysis}

Plant total RNAs were isolated using the RNA plant Plus Reagent Kit (Tiangen, Beijing, China) and TRIzol reagent (Invitrogen, Carlsbad, CA, USA). Reverse transcription was performed using the PrimeScript ${ }^{\mathrm{TM}}$ RT reagent Kit with the gDNA Eraser (TaKaRa, Shiga, Japan). The quantitative RT-PCR assay was carried out using the Step One Plus ${ }^{\mathrm{Tm}}$ Real-Time PCR System (Thermo Fisher Scientific, Waltham, MA, USA) to examine the transcription level of the wax and disease resistance-related genes. The qRT-PCR was performed according to [47]. The primers used for qRT-PCR are listed in Additional file 1: Table S3.

\section{Chlorophyll leaching assay}

The rosettes from 4-week-old seedlings were sampled. Chlorophyll content was determined following methods described previously [48, 49].

\section{Toluidine blue staining}

Toluidine blue staining was performed to detect leaf surface permeability, and carried out according to [45].

\section{Pathogen infection assays}

Leaves of WT and transgenic Arabidopsis were sampled and infected as described previously [50]. The Arabidopsis infection analysis with the bacterial strain Pseudomonas syringae pv tomato DC3000 (Pst DC3000) was carried out as described by [51]. Pst DC3000 is a model bacterial strain for the interaction between Arabidopsis and pathogenic bacteria. The inoculation of apple calli with $B$. dothidea was performed according to [50].

\section{ROS assays}

$\mathrm{H}_{2} \mathrm{O}_{2}$ was quantified according to the method described by [52]. Nitroblue tetrazolium (NBT) and 3,3-diaminobenzidine $(\mathrm{DAB})$ staining methods were applied to determine $\mathrm{O}_{2}{ }^{-}$and $\mathrm{H}_{2} \mathrm{O}_{2}$ levels, respectively $[50,53]$.

\section{Callose staining}

Four days after infecting with Pst DC3000, the leaves were rinsed $20 \mathrm{~h}$ in fixative solution (formaldehyde: acetic acid: ethanol: $\mathrm{H}_{2} \mathrm{O}, 3.7 \%$ : $5 \%: 50 \%: 41.3 \%$, by vol.). The sample was removed from the fixative and soaked with $8 \mathrm{M} \mathrm{NaOH}$ for $5-6 \mathrm{~h}$; the $\mathrm{NaOH}$ was removed and the sample was washed with deionized water for $10 \mathrm{~min}$; the water was removed, and $0.01 \%(\mathrm{w} / \mathrm{v})$ aniline blue dye solution was added in the dark for $1 \mathrm{~h}$. The quantitative statistics of the callose deposits was carried out using ImageJ software as described previously [50]. 


\section{Construction of the viral vectors and agroinfiltration of apple fruit}

Viral vectors were used as described by [54]. The primer pairs MdMYB30-F (5'-ATGGGGAGGCCTCCTTGCT$\left.3^{\prime}\right) /-\mathrm{R}$ (5' - GAAAAAGTTAGCATTTCCATGATCT3') were used to amplify the full-length MdMYB30, which was inserted into the pIR vector to generate the pIR-MdMYB30 construct (Additional file 1: Table S3). Then, a portion of the full-length cDNA of MdMYB30 (15-348 bp) was cloned and inserted into the tobacco rattle virus (TRV) vector in an antisense orientation to obtain the antisense construct TRV-MdMYB30. The instant injection method was used as described by [55]. After the injection, the fruit was placed in the dark for 3 days at room temperature, after which Botryosphaeria dothidea was inoculated at the injection point with an inoculating loop, placed in the dark at room temperature under humid conditions, and the proliferation of $B$. dothidea was observed after 3 days.

\section{Statistical analysis}

Each experiment was set up with three times repetitions and the data are based on the results of three parallel experiments. The data were analyzed for significance using Data Processing System (DPS) (http://www.chinadps. net/). Tukey's single factor test was used. All datasets were analyzed in the same way. A $p$-value $<0.05$ was considered significant.

\section{Additional file}

Additional file 1: Figure S1. Schematic diagram of MdMYB30 genomic and CDNA sequences. UTR: untranslated region. Figure S2. Changes in wax load, composition, and cuticular wax crystal morphology of WT plants and MdMYB30 ectopic expression plants detected by scanning electron microscopy. a Total cuticular wax content of leaves, calculated per unit area of 6-week-old Arabidopsis from the WT and MdMYB30 ectopic expression lines. b Cuticular wax composition of alkanes, alcohols, aldehydes, fatty acids, ketones, and esters on the stem surfaces of WT and MdMYB30 ectopic expression plants analyzed by GC-MS. Wax constituents are grouped by carbon chain length and chemical class. c Wax crystal morphology of 6-week-old Arabidopsis leaves from the WT and MdMYB30 ectopic expression lines (scale bars: $10 \mu \mathrm{m}$ ). Error bars represent standard deviation $(\mathrm{SD} ; n=3)$. Data are mean $\pm \mathrm{SD}$ of three independent replicates. Different lowercase letters indicate significant differences at $P<0.05$. Figure S3. Diagram representing the genomic structure and primer sets (indicated by P1-P3) analyzed in the MdKCS1 genes by ChIP-qPCR. White boxes represent primer sets, and black boxes represent ATG starting open reading frame (ORF). Table S1. Total epicuticular wax on wild type Arabidopsis, MdMYB30 OE4, OE5, and OE6 surface areas. Table S2. Epicuticular wax component $\left(\mu \mathrm{g} / \mathrm{dm}^{2}\right)$ in stems of wild-type Arabidopsis, MdMYB30 OE4, OE5, and OE6. Table S3. Primers used in this study. File S1. The amino acid sequences of MdMYB30 and homologs from 12 other plant species to analyze the phylogenetic relationships. (ZIP $1525 \mathrm{~kb}$ )

\section{Abbreviations}

B. dothidea: Botryosphaeria dothidea; ORF: Open reading frame; Pst DC3000: Bacterial strain Pseudomonas syringae pv tomato; ROS: Reactive oxygen species; SEM: Scanning electron microscopy; VLCFAs: Very-long-chain fatty acids

\section{Acknowledgements}

We would like to thank Prof. Takaya Moriguchi of the National Institute of Fruit Tree Science, Japan, for 'Orin' apple calli.

\section{Authors' contributions}

YYL and YJH initiated and designed the research. YLZ, CLZ, GLW, YXW, CHQ, QZ and $C X Y$ performed the experiments. YLZ analyzed the data. YYL contributed reagents/materials/analysis tools. YLZ wrote the manuscript. YYL and YLZ revised the manuscript. All authors have read and approved the manuscript.

\section{Funding}

This study was financially supported by the National Key Research and Development Program (2018YFD1000200), the National Natural Science Foundation of China (31772275), and the Natural Science Fund for Excellent Young Scholars of Shandong Province (ZR2018JL014). The funders had no role in the design of the study and collection, analysis, and interpretation of data and in writing the manuscript.

\section{Availability of data and materials}

All the data about the present study has been included in the table and/or figure form in the current manuscript or the supplement already. Authors are pleased to share analyzed/raw data and plant materials upon reasonable request.

\section{Ethics approval and consent to participate}

Not applicable.

\section{Consent for publication}

Not applicable.

\section{Competing interests}

The authors declare that they have no competing interests.

Received: 8 March 2019 Accepted: 2 July 2019

Published online: 19 August 2019

\section{References}

1. Beisson F, Li-Beisson Y, Pollar M. Solving the puzzles of cutin and suberinpolymer biosynthesis. Curr Opin in Plant Biol. 2012;15(3):329-37.

2. Qi CH, Zhao XY, Jiang H, Zheng PF, Liu HT, Li YY, Hao YJ. Isolation and functional identification of an apple MdCER1 gene. Plant Cell Tiss Org. 2018; 136(1):1-13.

3. Beattie GA, Marcell LM. Effect of alterations in cuticular wax biosynthesis on the physicochemical properties and topography of maize leaf surfaces. Plant Cell Environ. 2002;25(1):1-16.

4. Eigenbrode SD. The effects of plant epicuticular waxy blooms on attachment and effectiveness of predatory insects. Arthropod Struct Dev. 2004;33(1):91-102.

5. Raffaele S, Vailleau F, Léger A, Joubès J, Miersch O, Huard C, HuardC BE, Mongrand S, Domergue F, Roby D. A MYB transcription factor regulates very-long-chain fatty acid biosynthesis for activation of the hypersensitive cell death response in Arabidopsis. Plant Cell. 2008;20(3):752-67.

6. Stracke R, Werber M, Weisshaar B. The R2R3-MYB gene family in Arabidopsis thaliana. Curr Opin Plant Boil. 2001;4(5):447-56.

7. Glover BJ, Perez-Rodriguez M, Martin C. Development of several epidermal cell types can be specified by the same MYB-related plant transcription factor. Develop. 1998;125(17):3497-508.

8. Sugimoto K, Shin T, Hirohiko H. MYB-related transcription factor NtMYB2 induced by wounding and elicitors is a regulator of the tobacco retrotransposon Tto1 and defense-related genes. Plant Cell. 2000;12(12):2511-27.

9. Mengiste T, Chen X, Salmeron J, Dietrich R. The BOTRYTIS SUSCEPTIBLE1 gene encodes an R2R3 MYB transcription factor protein that is required for biotic and abiotic stress responses in Arabidopsis. Plant Cell. 2003;15(11): 2551-65.

10. Lee SB, Suh MC. Cuticular wax biosynthesis is up-regulated by the MYB94 transcription factor in Arabidopsis. Plant Cell Physiol. 2014;56(1):48-60.

11. Vailleau F, Daniel X, Tronchet M, Montillet JL, Triantaphylides C, Roby D. A R2R3-MYB gene, AtMYB30, acts as a positive regulator of the hypersensitive cell death program in plants in response to pathogen attack. Proc Natl Acad Sci U S A. 2002;99(15):10179-84. 
12. Kishi-Kaboshi M, Seo S, Takahashi A, Hirochika H. The MAMP-responsive MYB transcription factors MYB30, MYB55 and MYB110 activate the HCAA synthesis pathway and enhance immunity in Rice. Plant Cell Physiol. 2018;59(5):903-15.

13. Kaho M, Hiromasa M, Tomotaka I, Takamasa S, Mika N, Satomi S, Atsushi M, Tetsuya H, Yasuomi T, Wolfgang B, Hironaka T. MYB30 links ROS signaling, root cell elongation, and plant immune responses. Proc Natl Acad Sci U S A. 2018;115(20):E4710-9.

14. Saladié M, Matas AJ, Isaacson T, Jenks MA, Goodwin SM, Niklas KJ, Fernie A, Shackel K, Labavitch J, Xiaolin R, Lytovchenko A. A reevaluation of the key factors that influence tomato fruit softening and integrity. Plant Physiol. 2007;144(2):1012-28

15. Kerstin K, Hans-Jürgen E. The hydrophobic coatings of plant surfaces: Epicuticular wax crystals and their morphologies, crystallinity and molecular self-assembly. Micron. 2008;39:759-72.

16. Matas AJ, Cobb ED, Bartsch JA, Paolillo DJ, Niklas KJ. Biomechanics and anatomy of Lycopersicon esculentum fruit peels and enzyme-treated samples. Am J Bot. 2004;91:352-60.

17. Isaacson T. Cutin deficiency in the tomato fruit cuticle consistently affects resistance to microbial infection and biomechanical properties, but not transpirational water loss. Plant J. 2009;60(2):363-77.

18. Zong XJ, Li DP, Gu LK, Li DQ, Liu LX, Hu XL. Abscisic acid and hydrogen peroxide induce a novel maize group C MAP kinase gene, ZmMPK7, which is responsible for the removal of reactive oxygen species. Planta. 2009;229(3):485.

19. Lescot $M$, Déhais $P$, Thijs $G$, Marchal $K$, Moreau $Y$, Peer $W$, Rouzé $P$, Rombauts S. PlantCARE, a database of plant cis-acting regulatory elements and a portal to tools for in silico analysis of promoter sequences. Nucleic Acids Res. 2002;30(1):325-7.

20. Raffaele S, Rivas S. Regulate and be regulated: integration of defense and other signals by the AtMYB30 transcription factor. Front Plant Sci. 2013;4:98.

21. Jin HL, Martin C. Multifunctionality and diversity within the plant MYB-gene family. Plant Mol Biol. 1999;41(5):577-85.

22. Abe H, Urao T, Ito T, Seki M, Shinozaki K, Yamaguchi-Shinozaki K. Arabidopsis AtMYC2 (bHLH) and AtMYB2 (MYB) function as transcriptional activators in abscisic acid signaling. Plant Cell. 2003;15(1):63-78.

23. Lee SB, Kim H, Kim RJ, Suh MC. Overexpression of Arabidopsis MYB96 confers drought resistance in Camelina sativa via cuticular wax accumulation. Plant Cell Rep. 2014;33(9):1535-46.

24. Zhu L, Guo J, Zhu J, Zhou C. Enhanced expression of EsWAX1 improves drought tolerance with increased accumulation of cuticular wax and ascorbic acid in transgenic Arabidopsis. Plant Physiol Biochem. 2014;75:24-35.

25. Chen RG, Ni ZF, Nie XL, Qin YX, Dong GQ, Sun QX. Isolation and characterization of genes encoding Myb transcription factor in wheat (Triticum aestivem L.). Plant Sci. 2005;169(6):1146-54.

26. Qi TC, Huang H, Song SS, Xie DX. Regulation of jasmonate-mediated stamen development and seed production by a bHLH-MYB complex in Arabidopsis. Plant Cell. 2015;27(6):1620-33.

27. Feng K, Liu JX, Duan AQ, Li T, Yang QQ, Xu ZS, Xiong AS. AgMYB2 transcription factor is involved in the regulation of anthocyanin biosynthesis in purple celery (Apium graveolens L.). Planta. 2018;248(5):1249-61.

28. Yao LM, Jiang Y, Lu XX, Wang B, Zhou P, Wu TL. A R2R3-MYB transcription factor from Lablab purpureus induced by drought increases tolerance to abiotic stress in Arabidopsis. Mol Biol Rep. 2016;43(10):1089-00.

29. Bourdenx B, Bernard A, Domerque F, Stéphanie $P$, Amandine L, Dominique $R$, Marjorie $P$, Denis $V$, Richard PH, Johnathan AN, René L, Jérôme J. Overexpression of Arabidopsis ECERIFERUM1 promotes wax very-long-chain alkane biosynthesis and influences plant response to biotic and abiotic stresses. Plant Physiol. 2011;156(1):29-45.

30. Wang TY, Xing JW, Liu X, Yao YY, Hu Z, Peng H, Ni Z. GCN5 contributes to stem cuticular wax biosynthesis by histone acetylation of CER3 in Arabidopsis. J Exp Bot. 2018;69(12):2911-22.

31. Jenks MA, Rashotte AM, Tuttle HA, Feldmann KA. Mutants in Arabidopsis thaliana altered in epicuticular wax and leaf morphology. Plant Physiol. 1996;110(2):377-85.

32. Bessire $M$, Chassot $C$, Jacquat AC, Humphry M, Borel S, Petétot JCM, Nawrath C. A permeable cuticle in Arabidopsis leads to a strong resistance to Botrytis cinerea. EMBO J. 2007;26(8):2158-68.

33. Wang W, Huang Q, Liu Y, Sepehrnoori K. Experimental study on mechanisms of wax removal during pipeline pigging. In SPE Annual Technical Conference and Exhibition. Soc Pet Eng. 2015;9.

34. Wang $Y$, Wan $L$, Zhang $L$, Zhang Z, Zhang H, Quan R, Huang R. An ethylene response factor OsWR1 responsive to drought stress transcriptionally activates wax synthesis related genes and increases wax production in rice. Plant Mol Biol. 2012;78(3):275-88.

35. Pil JS, Saet BL, Mi CS, Mi-Jeong P, Young SG, Chung-Mo P. The MYB96 transcription factor regulates cuticular wax biosynthesis under drought conditions in Arabidopsis. Plant Cell. 2011;23(3):1138-52.

36. Radwan KA, Fayez SY, Mahmoud GL. Modifications of antioxidant activity and protein composition of bean leaf due to bean yellow mosaic virus infection and salicylic acid treatments. Acta Physiol Planta. 2010;32(5):891-904.

37. Peleg-Grossman S, Melamed-Book N, Levine A. ROS production during symbiotic infection suppresses pathogenesis-related gene expression. Plant Signal Behav. 2012;7:409-15.

38. Denness L. Cell wall damage-induced lignin biosynthesis is regulated by a reactive oxygen species- and jasmonic acid-dependent process in Arabidopsis. Plant Physiol. 2011;156:1364-74.

39. Zhang YL, Zhang CL, Wang GL, Wang YX, Qi CH, You CX, Li YY, Hao YJ. Apple AP2/EREBP transcription factor MdSHINE2 confers drought resistance by regulating wax biosynthesis. Planta. 2019;249(5):1627-43.

40. Zhang CL, Mao K, Zhou L, Wang GL, Zhang YL, Li YY, Hao YJ. Genomewide identification and characterization of apple long-chain acyl-CoA synthetases and expression analysis under different stresses. Plant Physiol Biotech. 2018;132:320-32.

41. Liu X, Liang Y, Zhang W, Huo Y, Feng S, Qiu H, He X, Wu S, Chen X. MdFLS2 recognizes bacterial flagellin flg22 and enhances immune resistance against apple ring rot causal fungi in Arabidopsis fls2 mutant. Acta Horticulturae Sinica. 2018;45(5):827-44.

42. An JP, Li HH, Song LQ, Su L, Liu X, You CX, Hao YJ. The molecular cloning and functional characterization of MdMYC2, a bHLH transcription factor in apple. Plant Physiol Bioch. 2016;108:24-31.

43. Clough SJ, Bent AF. Floral dip: a simplified method for agrobacteriummediated transformation of Arabidopsis thaliana. Plant J. 1998;16(6):735-43.

44. Sun MH, Ma QJ, Hu DG, Zhu XP, You CX, Shu HR, Hao YJ. The glucose sensor MdHXK1 phosphorylates a tonoplast $\mathrm{Na}^{+} / \mathrm{H}^{+}$exchanger to improve salt tolerance. Plant Physiol. 2018:176(4):2977-90.

45. Aharoni A, Dixit S, Jetter R, Thoenes E, van Arkel G, Pereira A. The SHINE clade of AP2 domain transcription factors activates wax biosynthesis, alters cuticle properties, and confers drought tolerance when overexpressed in Arabidopsis. Plant Cell. 2004;16(9):2463-80.

46. An JP, Zhang XW, Bi SQ, You CX, Wang XF, Hao YJ. MdbHLH93, an appleactivator regulating leaf senescence, is regulated by ABA and MdBT2 in antagonistic ways. New Phytol. 2019;222(2):735-51.

47. An JP, Zhang XW, Xu RR, You CX, Wang XF, Hao YJ. Apple MdERF4 negatively regulates salt tolerance by inhibiting MdERF3 transcription. Plant Sci. 2018;276:181-8.

48. Wang ZY, Tian XJ, Zhao QZ, Liu ZQ, Li XF, Ren YK, Tang JQ, Fang J, Xu QI, $\mathrm{Bu}$ QY. The E3 ligase DROUGHT HYPERSENSITIVE negatively regulates cuticular wax biosynthesis by promoting the degradation of transcription factor ROC4 in rice. Plant Cell. 2018;30(1):228-44.

49. Lolle SJ, Berlyn GP, Engstrom EM, Krolikowski KA, Reiter WD, Pruitt RE. Developmental regulation of cell interactions in the Arabidopsis fiddlehead-1Mutant: a role for the epidermal cell wall and cuticle. Dev boil. 1997;189(2):311-21.

50. Han PL, Dong YH, Gu KD, Yu JQ, Hu DG, HaoYJ. The apple U-box E3 ubiquitin ligase MdPUB29 contributes to activate plant immune response to the fungal pathogen Botryosphaeria dothidea. Planta. 2019;249(4):1177-88.

51. Xin XF, Sheng YH. Pseudomonas syringae pv. tomato DC3000: a model pathogen for probing disease susceptibility and hormone signaling in plants. Annu Rev Phytopathol. 2013;51:473-98.

52. Zhang H, Liu Y, Wen F, Yao D, Wang L, Guo J. A novel rice C2H2-type zinc finger protein, ZFP36, is a key player involved in abscisic acid-induced antioxidant defence and oxidative stress tolerance in rice. J Exp Bot. 2014;65:5795-809.

53. Zhao H, Xu L, Su T, Jiang Y, Hu L, Ma F. Melatonin regulates carbohydratemetabolism and defenses against Pseudomonas syringae pv. tomato DC 3000 infection in Arabidopsis thaliana. J Pineal Res. 2015;59(1):109-19.

54. Li L, Lomonossoff GP. Agroinfection as a rapid method for propagating Cowpea mosaic virus-based constructs. J Virological Methods. 2002;105(2):343-8.

55. Li YY, Mao K, Zhao C, You CX, Hao YJ. MdCOP1 ubiquitin E3 ligases interact with MdMYB1 to regulate light-induced anthocyanin biosynthesis and red fruit coloration in apple. Plant Physiol. 2012;160(2):1011-22.

\section{Publisher's Note}

Springer Nature remains neutral with regard to jurisdictional claims in published maps and institutional affiliations. 